\title{
Usefulness of Diffusion Weighted Imaging and Apparent Diffusion Coefficient in Characterizing Pancreatitis and Pancreatic Adenocarcinoma
}

\author{
Prabhu Radhan ${ }^{1}$, Anupama Chandrasekharan², Rajoo Ramachandran³, Remya Baburaj, Poovini Soundararajan ${ }^{5}$ \\ ${ }^{1}$ Department of Radio Diagnosis, Sri Ramachandra Institute of Higher Education and Research, Chennai, Tamilnadu, \\ India. ${ }^{2}$ Department of Radio Diagnosis, Sri Ramachandra Institute of Higher Education and Research, Chennai, \\ Tamilnadu, India. ${ }^{3}$ Department of Radio Diagnosis, Sri Ramachandra Institute of Higher Education and Research, \\ Chennai, Tamilnadu, India. ${ }^{4}$ Department of Radio Diagnosis, Sri Ramachandra Institute of Higher Education and \\ Research, Chennai, Tamilnadu, India. ${ }^{5}$ Department of Radio Diagnosis, Sri Ramachandra Institute of Higher Education \\ and Research, Chennai, Tamilnadu, India.
}

\section{ABSTRACT}

\section{BACKGROUND}

The main purpose of this article is to establish the usefulness of Diffusion Weighted Imaging (DWI) and Apparent Diffusion Coefficient (ADC) as complementary tools to conventional MRI sequences in characterizing benign (pancreatitis) and malignant (pancreatic adenocarcinoma) pancreatic pathologies in comparison to a control group.

\section{METHODS}

A prospective observational study was done over a period of two years with images obtained using Siemens Magnatom Avanto 1.5T MRI. 130 patients with pancreatic abnormalities were included in this study. An additional control group included 32 patients with normal pancreatic parenchyma. MRI findings including DWI and ADC values were calculated for all patients which was then correlated with clinical, lab and histopathological data.

\section{RESULTS}

Out of the 130 positive cases, 116 (89 \%) were benign and 14 (11\%) were malignant lesions based on MRI features. Benign lesions include acute pancreatitis (47\%), acute on chronic pancreatitis (11\%) and chronic pancreatitis (31\%). Rest of the cases were pancreatic adenocarcinomas (11\%). There was significant reduction of ADC values in patients with acute pancreatitis (mean ADC - $1.016 \times 10^{-3}$ ), acute on chronic pancreatitis (mean ADC - $1.122 \times 10^{-3}$ ) and chronic pancreatitis (mean ADC - $1.277 \mathrm{x}$ $10^{-3}$ ) as well as pancreatic carcinoma (mean ADC - $0.934 \times 10^{-3}$ ) in comparison with the control group (mean ADC - $1.363 \times 10^{-3}$ ).

\section{CONCLUSIONS}

DWI with ADC mapping can play a significant role in the evaluation of pancreatic diseases. It can help in differentiating benign and malignant disease (ADC values of malignancy are significantly lower than benign disease).

\section{KEY WORDS}

Pancreatitis, DWI, MRI, ADC, Adenocarcinoma

\author{
Corresponding Author: \\ Dr. Poovini Soundararajan, \\ 7/4, Logaya Colony, $4^{\text {th }}$ Cross Street, \\ Saligramam, Chennai-600093, \\ Tamilnadu, India. \\ E-mail:spoovini@gmail.com
}

DOI: $10.14260 / j e m d s / 2019 / 833$

Financial or Other Competing Interests: None.

How to Cite This Article:

Radhan $P$, Chandrasekharan A, Ramachandran $R$, et al. Usefulness of diffusion weighted imaging and apparent diffusion coefficient in characterizing pancreatitis and pancreatic adenocarcinoma. J. Evolution Med. Dent. Sci. 2019;8(51):3846-3849, DOI: $10.14260 /$ jemds/2019/833

Submission 12-11-2019,

Peer Review 10-12-2019,

Acceptance 13-12-2019,

Published 23-12-2019. 


\section{BACKGROUND}

Diffusion Weighted Imaging (DWI) and Apparent Diffusion Coefficient (ADC) are functional imaging tools in Magnetic Resonance Imaging (MRI) that help define tissue characteristics on the basis of random Brownian movement of water molecules. DWI provides qualitative information about the cellularity of the tissues and helps in differentiating abnormal tissue from normal tissue while ADC is a quantitative entity derived from DWI.

Although DWI was initially widely used only in neuroimaging, subsequent advances in MR imaging software and hardware such as "ultrafast" MR imaging techniques and multicoil receiver systems have made diffusion weighted (DW) MR imaging a practicable tool in imaging of abdominal organs such as pancreas overcoming initial limitations related to image artefacts. ${ }^{[1]}$

In general, restricted diffusion and lower ADC values are seen in highly cellular tumours, fibrotic tissue, abscess and cytotoxic oedema. Conversely, tissues with low cellularity \& disrupted membranes show fast unrestricted movement of water molecules with facilitated diffusion and high ADC values.[2]

Though ultrasound is the first-line imaging modality used in imaging patients with abdominal complaints, sonographic imaging of pancreas can sometimes be challenging or even suboptimal due to factors like bowel gas shadows and obesity because of the inherent retroperitoneal location of pancreas. Hence, cross sectional imaging plays a major role in detecting pancreatic pathologies and the use of MRI for diagnosis and further characterization of pancreatic diseases is increasing day by day.[3] Our aim was to study the DWI and ADC values of benign and malignant pancreatic pathologies in comparison to a control group and therefore assess the utility of DWI and ADC as complementary tools to conventional MR sequences in diagnosing and classifying pancreatic pathologies.

\section{METHODS}

This prospective observational study was done over a period of two years after obtaining clearance from the institutional ethics committee. The images were obtained using Siemens Magnatom Avanto 1.5 T MRI. The study group included 130 patients with pancreatic abnormalities and an additional control group of 32 patients with normal pancreatic parenchyma. MRI findings including DWI and ADC values were studied to differentiate benign and malignant lesions which were further correlated with clinical, lab and histopathological data.

\section{MRI Protocol}

Diffusion weighted images were obtained in the axial plane using a single-shot echo planar imaging sequence (TR - 1000 ms/TE - $74 \mathrm{~ms}$; slice thickness $6 \mathrm{~mm}$; FOV 36-42 cm; matrix, $384 \mathrm{x} 25$, b-value of $1,000 \mathrm{~s} / \mathrm{mm}^{2}$ ). Conventional MRI images and DWI imaging data including ADC values were then analysed.

\section{Image Analysis}

Following the generation of ADC map in grey scale, the Region of interest (ROI) was placed in areas of true restriction without necrosis or haemorrhage. Two ROI of size $50+/-10 \mathrm{~mm}^{2}$ were placed following which ADC was computed automatically by the pre-set software (Argus in Siemens). Mean ADC was then calculated by averaging of the ADC values. The findings on Conventional MRI were correlated with findings on DWI (with corresponding $\mathrm{ADC}$ values). The mean ADC values were compared between the groups using $\mathrm{N}$ par Mann-Whitney test.

\section{RESULTS}

During the study period, a total of 130 cases with pancreatic pathologies were identified on MRI abdomen / MRCP with a wide range of age of presentation ranging from 12 to 93 years. Among these, males constituted $64 \%$ of the study population. Additionally, normalized ADC values were calculated for a control group of 32 patients who were free of pancreatic disease after averaging ADC values of head, body and tail. The mean ADC value of this control group was found to be 1.36326 $\times 10^{-3} \mathrm{~mm}^{2 /} / \mathrm{s}$.

Out of the 130 positive cases, based on MRI features 116 (89\%) cases were classified as benign and 14 (11\%) were classified as malignant lesions. The benign lesions included were acute pancreatitis (47\%), acute on chronic pancreatitis $(11 \%)$ and chronic pancreatitis $(31 \%)$. Rest of the cases were pancreatic adenocarcinomas (11\%). As anticipated, all the above mentioned pathologies demonstrated hyperintense signal on DWI images, following which ADC values were then calculated from areas of true diffusion. The mean ADC value in the control group and the various pancreatic pathologies were charted as shown in Table 1.

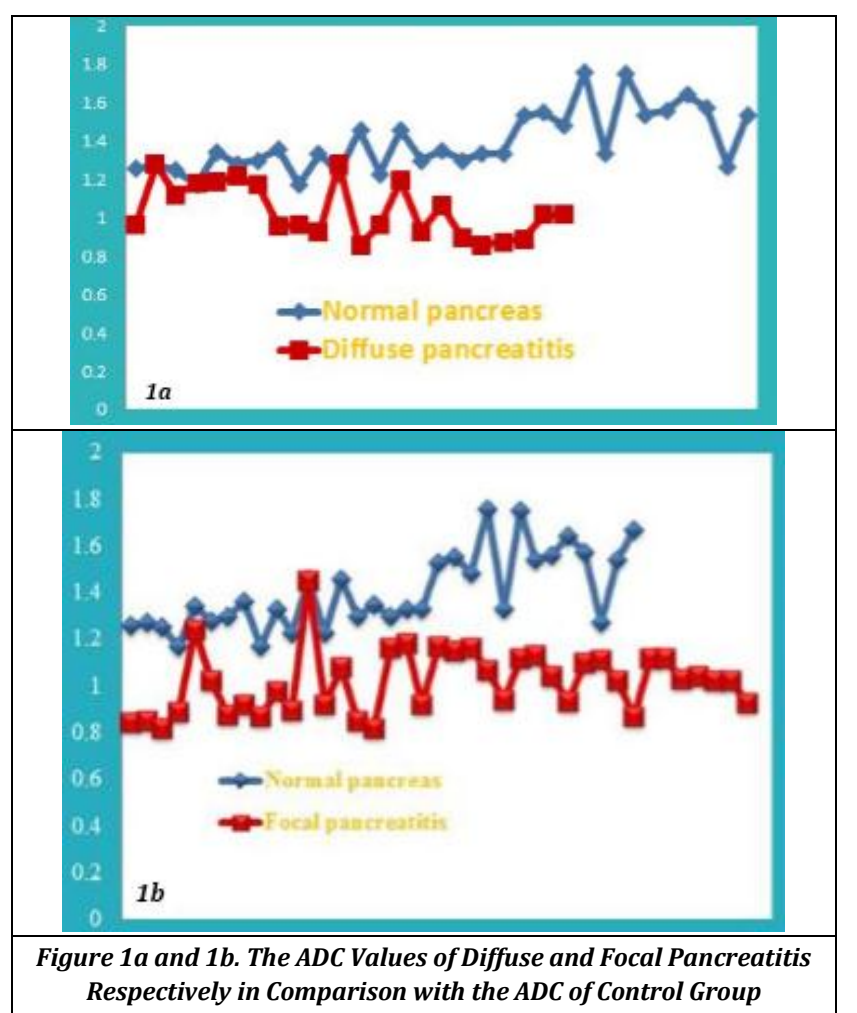



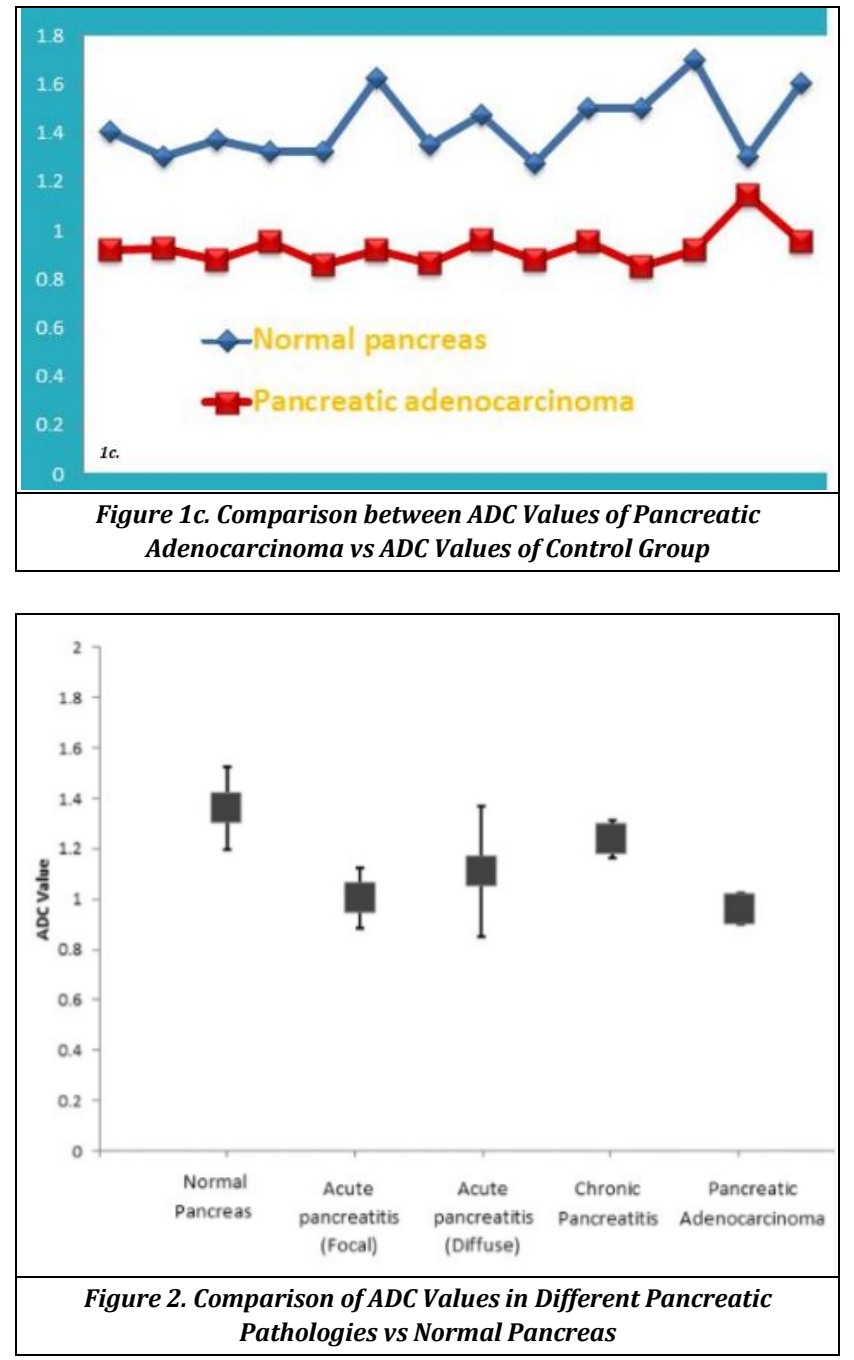

\begin{tabular}{|c|c|c|c|c|c|}
\hline \multicolumn{7}{|c|}{ Descriptive Statistics } \\
\hline & $\mathbf{N}$ & $\begin{array}{c}\text { Minimum } \\
\left(\times \mathbf{1 0}^{-3}\right. \\
\left.\mathbf{m m}^{2} / \mathbf{s}\right)\end{array}$ & $\begin{array}{c}\text { Maximum } \\
\left(\times \mathbf{1 0}^{-3}\right. \\
\left.\mathbf{m m}^{2} / \mathbf{s}\right)\end{array}$ & $\begin{array}{c}\text { Mean } \\
\left(\times \mathbf{1 0}^{-3}\right. \\
\left.\mathbf{m m}^{2} / \mathbf{s}\right)\end{array}$ & S.D. \\
\hline Normal Pancreas & $\mathbf{3 2}$ & $\mathbf{1 . 1 7}$ & $\mathbf{1 . 7 6 3 3}$ & $\mathbf{1 . 3 6 3 2 6}$ & $\mathbf{0 . 1 6 4 2 9 4}$ \\
\hline Focal Pancreatitis & 39 & 0.818 & 1.45 & 1.016512 & 0.138409 \\
\hline Diffuse acute Pancreatitis & 22 & 0.852 & 1.28 & 1.12239 & 0.342532 \\
\hline Acute on chronic Pancreatitis & 15 & 0.863 & 1.55 & 1.12333 & 0.163605 \\
\hline Chronic Pancreatitis & 40 & 0.745 & 1.566 & 1.277061 & 0.152202 \\
\hline Pancreatic adenocarcinoma & 14 & 0.85 & 1.14 & 0.934071 & 0.076194 \\
\hline Table 1. ADC Value of Normal Pancreas and Various Pancreatic \\
\hline \multicolumn{7}{|c|}{ Pathologies } \\
\hline
\end{tabular}

\section{DISCUSSION}

In our study, among the 130 patients imaged, diagnosis of acute interstitial oedematous pancreatitis was found in 61 patients which was then further classified into focal/segmental pancreatitis $(n=39)$ and diffuse pancreatitis $(n=22)$ on the basis of disease distribution.

The mean ADC values for focal and diffuse acute pancreatitis were found to be $1.0165 \times 10^{-3} \mathrm{~mm}^{2} / \mathrm{s} \pm 0.13$ and $1.323 \times 10^{-3} \mathrm{~mm}^{2} / \mathrm{s} \pm 0.34$ respectively (Figure $1 \mathrm{a}$ and $1 \mathrm{~b}$ ). These values were found to be significantly lower $(\mathrm{p}<0.0005)$ than the mean ADC value of the control group which was $1.36326 \times 10^{-3} \mathrm{~mm}^{2} / \mathrm{s} \pm 0.16$. Moreover, in 7 patients with focal pancreatitis, although conventional MR sequence images were found to be normal, segmental increased signal on DWI images facilitated the diagnosis of focal / segmental pancreatitis, as confirmed by elevated levels of serum amylase / lipase. Isolated head involvement was the predominant pattern in segmental pancreatitis accounting for $51 \%$ followed by the tail region (30\%).

Our findings correlated with a study by Yencilek E et al conducted with a smaller study population which also found that " the mean pancreatic ADC values in acute pancreatitis $\left(1.19 \times 10(-3) \mathrm{mm}^{2} / \mathrm{sn} \pm 0.32\right)$ was significantly lower than in the normal group $\left(1.78 \times 10(-3) \mathrm{mm}^{2} / \mathrm{sn} \pm 0.29\right)(\mathrm{p}<0.001)$ " $[4]$ It is hypothesized that the decrease in ADC values in acute pancreatitis is multifactorial occurring secondary to acinar cell death and subsequent pathological changes such as intercellular fibrin deposition. ${ }^{[5]}$

Additionally, in our study, ADC values were also found to be lower than the control group in 15 patients with acute on chronic pancreatitis with a mean of $1.122 \times 10^{-3} \mathrm{~mm}^{2} / \mathrm{s} \pm 0.14$.

In 40 patients diagnosed with chronic pancreatitis ADC values ranged from $0.745 \times 10^{-3} \mathrm{~mm}^{2} / \mathrm{s}$ to $1.566 \times 10^{-3} \mathrm{~mm}^{2} / \mathrm{s}$ with a mean value of $1.277 \times 10^{-3} \mathrm{~mm}^{2} / \mathrm{s}$. The overlap with control group ADC values seen in few patients may be attributed to the complete resolution of inflammation. Although chronic pancreatitis usually results in diffuse atrophy of pancreatic parenchyma, it can occasionally cause focal enlargement of the gland, simulating a neoplasm. [6] In such cases, similar to acute focal pancreatitis, ADC values can serve as problem solving tools in addition to conventional MR sequences to distinguish mass- forming pancreatitis from a malignant mass lesion.

The importance of ADC values in diagnosis of malignancies of liver, breast, uterus, cervix have been widely studied.[7-10] Few similar studies have explored the limitations and diagnostic challenges faced while using DWI/ADC to detect pancreatic malignancies.[11,12] In our study, the ADC value in pancreatic malignancies $(n=14)$ ranged from $0.850 \times 10^{-3}$ $1.14 \times 10^{-3} / \mathrm{s}$ with a mean ADC value of $0.9340 \times 10^{-3} \mathrm{~mm}^{2} / / \mathrm{s} \pm 0.07$ which was found to be pointedly lower than the normal control group of $1.36326 \times 10^{-3} \mathrm{~mm}^{2}$ $\mathrm{s} \pm 0.16(\mathrm{p}<0.0005)$, (Figure $1 \mathrm{c})$ thus clearly defining the utility of DWI/ ADC in such cases. All cases of suspected malignancies were followed up and the imaging diagnosis was confirmed with correlation of histopathological findings.

Compared to the ADC values of benign pathologies like acute (focal/diffuse) pancreatitis and chronic pancreatitis, the ADC values in pancreatic malignancies were found to be significantly lower than the normal parenchyma (Figure 2). Statistical analysis obtained from 130 patients included in our study revealed significant $p$ value $(<0.0005)$ between ADC values in different subgroups of pancreatitis versus pancreatic adenocarcinoma.

As described above, DWI images also aided diagnosis of segmental pancreatitis by demonstrating focal hyperintense signal even in cases where the pancreatic parenchyma appeared apparently normal on conventional MR sequences.

Few limitations of DWI/ADC imaging include poor spatial resolution, lack of standardized imaging parameters/ protocols and questionable reproducibility of ADC values. [13] Additionally inevitable errors in ADC measurements can sporadically occur in body imaging due to MR artefacts despite optimization of imaging protocols. In patients with extensive parenchymal necrosis and in mass lesions with significant haemorrhage, T2 shine through confounds DWI images and accurate $\mathrm{ADC}$ values cannot be attained. Another inherent 
limitation of our study is that our results were obtained with 1.5 $\mathrm{T}$ scanner similar to few other studies on DWI/ADC in abdominal imaging.[5,14,15] Therefore the probability that results obtained with a MR machine of higher magnetic field (3 T) may differ cannot be ruled out.

\section{CONCLUSIONS}

In our study, although the various pancreatic pathologies show reduced ADC values compared to normal parenchyma, pancreatic malignancies were found to have significantly lower ADC values. DWI also served as a problem-solving tool in diagnosis of select cases of pancreatitis when conventional MR images were apparently normal. In conclusion, DWI/ADC play an important role in distinguishing benign from malignant pancreatic entities and serve as useful complementary tools to conventional MR sequences in diagnosing and classifying pancreatic pathologies.

\section{REFERENCES}

[1] Barral M, Taouli B, Guiu B, et al. Diffusion-weighted MR imaging of the pancreas: current status and recommendations. Radiology 2014;274(1):45-63.

[2] Qayyum A. Diffusion-weighted imaging in the abdomen and pelvis: concepts and applications. Radiographics 2009;29(6):1797-810.

[3] Chaudhary V, Bano S. Imaging of the pancreas: recent advances. Indian Journal of Endocrinology and Metabolism 2011;15(Suppl 1):S25-S32.

[4] Yencilek E, Telli S, Tekesin K, et al. The efficacy of diffusion weighted imaging for detection of acute pancreatitis and comparison of subgroups according to Balthazar classification. Turk J Gastroenterol 2014;25(5):553-7.

[5] Thomas S, Kayhan A, Lakadamyali H, et al. Diffusion MRI of acute pancreatitis and comparison with normal individuals using ADC values. Emergency Radiology 2012;19(1):5-9.
[6] Kim T, Murakami T, Takamura M, et al. Pancreatic mass due to chronic pancreatitis: correlation of CT and MR imaging features with pathologic findings. American Journal of Roentgenology 2001;177(2):367-71.

[7] Guo Y, Cai YQ, Cai ZL, et al. Differentiation of clinically benign and malignant breast lesions using diffusionweighted imaging. Journal of Magnetic Resonance Imaging 2002;16(2):172-8.

[8] Naganawa S, Sato C, Kumada H, et al. Apparent diffusion coefficient in cervical cancer of the uterus: comparison with the normal uterine cervix. European Radiology 2005;15(1):71-8.

[9] Fujii S, Matsusue E, Kigawa J, et al. Diagnostic accuracy of the apparent diffusion coefficient in differentiating benign from malignant uterine endometrial cavity lesions: initial results. European Radiology 2008;18(2):384-9.

[10] Jahic E, Sofic A, Selimovic AH. DWI/ADC in differentiation of benign from malignant focal liver lesion. Acta Informatica Medica 2016;24(4):244-7.

[11] Fukukura Y, Takumi K, Kamimura K, et al. Pancreatic adenocarcinoma: variability of diffusion-weighted MR imaging findings. Radiology 2012;263(3):732-40.

[12] Sandrasegaran K, Nutakki K, Tahir B, et al. Use of diffusion-weighted MRI to differentiate chronic pancreatitis from pancreatic cancer. American Journal of Roentgenology 2013;201(5):1002-8.

[13] Lin WC, Chen JH. Pitfalls and limitations of diffusionweighted magnetic resonance imaging in the diagnosis of urinary bladder cancer. Translational Oncology 2015;8(3):217-30.

[14] Matsuki M, Inada Y, Nakai G, et al. Diffusion-weighed MR imaging of pancreatic carcinoma. Abdominal Imaging 2007;32(4):481-3.

[15] De Freitas TF, Schraibman V, Ardengh JC, et al. Diffusionweighted magnetic resonance imaging indicates the severity of acute pancreatitis. Abdominal Imaging 2015;40(2):265-71. 